Aritoshi Iida - Susumu Saito - Akihiro Sekine

Yuri Kitamura - Kimie Kondo - Chihiro Mishima

Saori Osawa · Satoko Harigae · Yusuke Nakamura

\title{
High-density single-nucleotide polymorphism (SNP) map of the 150-kb region corresponding to the human ATP-binding cassette transporter A1 (ABCA1) gene
}

Received: May 22, 2001 / Accepted: June 12, 2001

\begin{abstract}
Highly dense catalogs of human genetic variations, in combination with high-throughput genotyping technologies, are expected to clarify individual genetic differences in pharmacological responsiveness and predispositions to common diseases. Here we report single-nucleotide polymorphisms (SNPs) present among 48 Japanese individuals at the locus for the human ATP-binding cassette transporter A1 ( $A B C A 1)$ gene. ABCA1 plays a key role in apolipoprotein-mediated cholesterol transport, and mutations in this gene are responsible for Tangier disease and familial high-density lipoprotein deficiency associated with reduced cholesterol efflux. We identified a total of 162 SNPs, 149 of which were novel, within the $150-\mathrm{kb}$ region encompassing the entire $A B C A 1$ gene. Eight of the SNPs lie within coding elements, two in $5^{\prime}$ flanking regions, 147 in introns, and five in $3^{\prime}$ untranslated regions, but none were found in $5^{\prime}$ untranslated or $3^{\prime}$ flanking regions. The ratio of transitions to transversions was approximately 2.37 to 1 . Our dense SNP map of this region could serve as a powerful resource for studies of complex genetic diseases that may be associated with $A B C A 1$ and of individual responses to drug therapy.
\end{abstract}

Key words Single-nucleotide polymorphism (SNP) Insertion-deletion polymorphism · High-density SNP map · ATP-binding cassette A1 transporter gene $\cdot$ Japanese population $\cdot$ Nonsynonymous substitution

A. Iida $\cdot$ S. Saito $\cdot$ A. Sekine $\cdot$ Y. Kitamura $\cdot$ K. Kondo $\cdot$ C. Mishima S. Osawa $\cdot$ S. Harigae $\cdot$ Y. Nakamura

Laboratory for Genotyping, RIKEN SNP Research Center, Tokyo, Japan

Y. Nakamura $(\bowtie)$

Laboratory of Molecular Medicine, Human Genome Center,

Institute of Medical Science, The University of Tokyo, 4-6-1

Shirokanedai, Minato-ku, Tokyo 108-8639, Japan

Tel. +81-3-5449-5372; Fax +81-3-5449-5433

e-mail: yusuke@ims.u-tokyo.ac.jp

\section{Introduction}

The human ATP-binding cassette transporter A1 (ABCA1) gene encodes a $247-\mathrm{kDa}$ membrane protein with two separate transmembrane portions, each consisting of six membrane-spanning domains and a nucleotide-binding fold. The regulatory domain contains a highly hydrophobic segment and is expressed on the plasma membrane and the Golgi complex (Luciani et al. 1994; Orso et al. 2000). Functional analyses of ABCA1 have revealed roles in (1) regulation of the apolipoprotein A1-dependent cellular export of cholesterol and phopholipids (Langmann et al. 1999; Oram 2000); (2) engulfment of apoptotic cells by macrophages (Luciani and Chimini 1996; Hamon et al. 2000); (3) secretion of macrophage interleukin-1 beta (Hamon et al. 1997); and (4) caveolar processing (Orso et al. 2000). The genomic sequence at the $A B C A 1$ locus that has been determined so far includes $1453 \mathrm{bp}$ of the promoter region, 146,581 bp of introns and exons, and $1000 \mathrm{bp}$ of DNA downstream of the polyadenylation signal (Santamarina-Fojo et al. 2000). The gene itself consists of 50 exons ranging from 33 to $245 \mathrm{bp}$. Relationships between $A B C A 1$ gene and human dyslipidemic diseases have been investigated intensively; mutations of $A B C A 1$ gene can be found in patients with Tangier disease and in persons with familial high-density lipoprotein (HDL) deficiency associated with reduced cholesterol efflux (Bodzioch et al. 1999; Brooks-Wilson et al. 1999; Marcil et al. 1999; Rust et al. 1999). Recently Clee et al. (2001) isolated 16 single-nucleotide polymorphisms (SNPs) from coding elements of the $A B C A 1$ gene and revealed that some are associated with altered plasma lipid levels and risk of coronary artery disease.

Variations in genes that encode drug-transporter and drug-metabolizing enzymes may be associated with susceptibility to common diseases and with differences in therapeutic efficacy and side effects of drugs among individual persons, because some variations can alter the activity of a gene product with respect to quality, quantity, or both. Therefore, information concerning naturally occurring genetic variants in human transporter genes such as $A B C A 1$ 


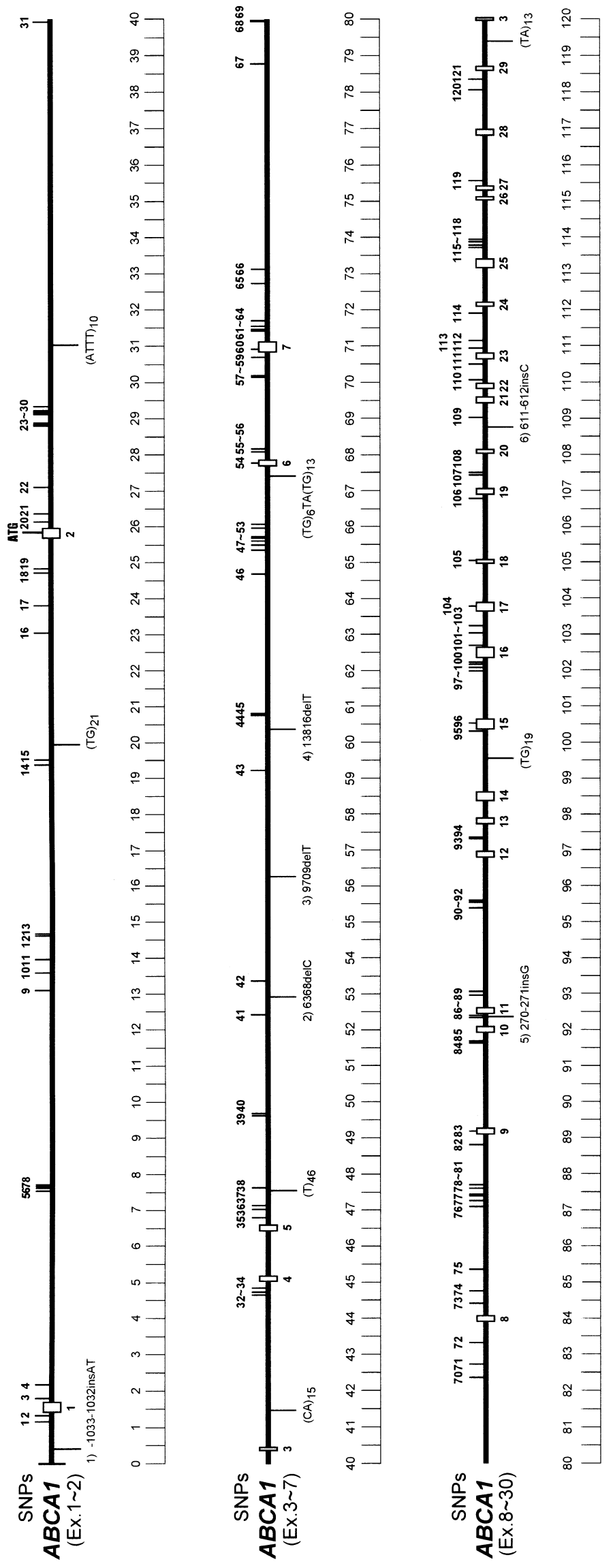

亏ิ

ฐ

$\cong$

sิ

○

สี

$\frac{0}{20}$

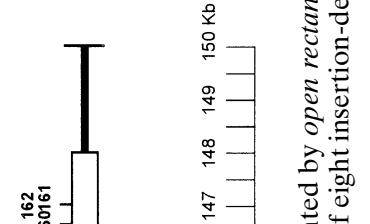
政-

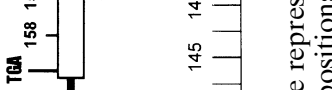

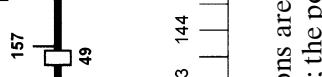

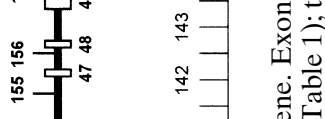

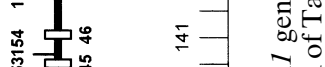

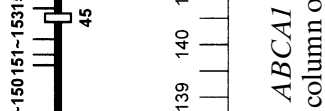
旁寻

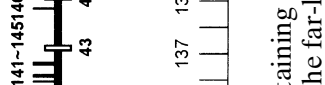

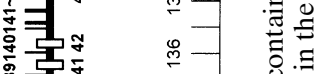

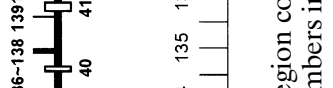

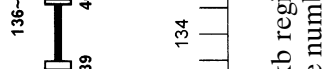

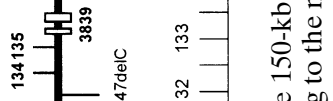
的然

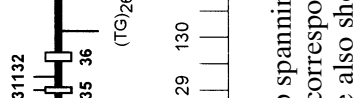

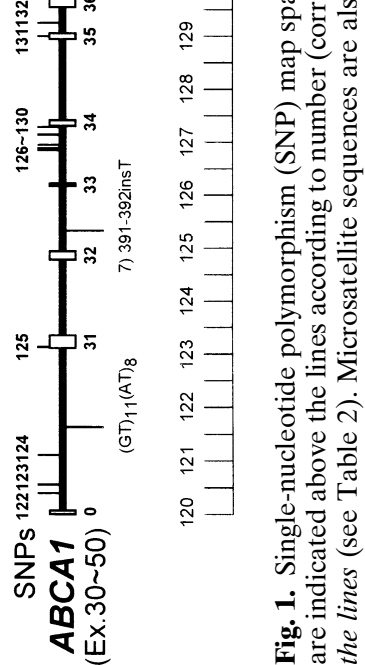


Table 1. Characterization of 162 single-nucleotide polymorphisms (SNPs) within the $A B C A 1$ locus

\begin{tabular}{|c|c|c|c|c|c|c|c|}
\hline Number & Location & Exon & $\operatorname{SNP}\left(5^{\prime}\right.$ to $\left.3^{\prime}\right)$ & Substituion & $\begin{array}{l}\text { Repetitive } \\
\text { sequence }\end{array}$ & $\begin{array}{l}\text { Identity } \\
\text { to dbSNP }\end{array}$ & Reference \\
\hline 1 & $-278 \mathrm{G}>\mathrm{C} 5^{\prime}$ flanking region & & gggcccgggcgggggaaggg $\mathrm{G} / \mathrm{C}$ acgcagaccgcggaccctaa & & & rs1800976 & \\
\hline 2 & $-99 \mathrm{G}>\mathrm{C} 5$ ' flanking region & & acataaacagaggccgggaa $\mathrm{G} / \mathrm{C}$ ggggcggggaggagggagag & & & & \\
\hline 3 & $159 \mathrm{G}>\mathrm{T}$ intron 1 & & gcggtgttaaatggggagac $\mathrm{G} / \mathrm{T}$ atgtcctagtacgagctctg & & & & \\
\hline 4 & $506 \mathrm{G}>\mathrm{C}$ intron 1 & & gaattggctatatgctccec $\mathrm{G} / \mathrm{C}$ ggactggagcggcacagtcc & & & & \\
\hline 5 & $5897 \mathrm{~T}>\mathrm{G}$ intron 1 & & gtacaaaaccctttagcttt T/G gcaaacctcctttaagaccc & & & & \\
\hline 6 & $5929 \mathrm{C}>\mathrm{T}$ intron 1 & & ttaagacccgatttaaatgc $\mathrm{C} / \mathrm{T}$ tccetcctcatgaagctctt & & & & \\
\hline 7 & $5962 \mathrm{~T}>\mathrm{C}$ intron 1 & & aagctcttctggatccactc $\mathrm{T} / \mathrm{C}$ ttcccatcactaagttgaaa & & & & \\
\hline 8 & $5985 \mathrm{~A}>\mathrm{C}$ intron 1 & & cccatcactaagttgaaagt $\mathrm{A} / \mathrm{C}$ agatcccettctcttactt & & & & \\
\hline 9 & $11416 \mathrm{G}>\mathrm{A}$ intron 1 & & ttacagtgccctttatagga $\mathrm{G} / \mathrm{A}$ agaaagaagaaattgtgtct & & & & \\
\hline 10 & $11935 \mathrm{G}>\mathrm{A}$ intron 1 & & tctctgtggagcaaatagag G/A gctgtctgacacttggttcc & & & & \\
\hline 11 & $12281 \mathrm{~T}>\mathrm{A}$ intron 1 & & gaatgtttgatttgtgaaaa $\mathrm{T} / \mathrm{A}$ cttaataacagtagttttt & & & & \\
\hline 12 & $12924 \mathrm{~T}>\mathrm{C}$ intron 1 & & gtgctgacaatcttatactc $\mathrm{T} / \mathrm{C}$ aggttgaacctccggggaag & & & & \\
\hline 13 & $13002 \mathrm{C}>\mathrm{G}$ intron 1 & & gagcctcaatcacagattct $\mathrm{C} / \mathrm{G}$ tctagctcacatgaagttaa & & & & \\
\hline 14 & $17715 \mathrm{C}>\mathrm{T}$ intron 1 & & ggagcatgactttgtggaag $\mathrm{C} / \mathrm{T}$ ctctcctcttccacccagag & & & & \\
\hline 15 & $17848 \mathrm{~T}>\mathrm{C}$ intron 1 & & gagggctgactgtcaccctt $\mathrm{T} / \mathrm{C}$ gataggagcccagcactaaa & & & & \\
\hline 16 & $21384 \mathrm{G}>\mathrm{C}$ intron 1 & & gtgggtgggaggaattggag $\mathrm{G} / \mathrm{C}$ aggaagcttgcctaagtgtg & & & & \\
\hline 17 & $22145 \mathrm{C}>\mathrm{G}$ intron 1 & & gtagcttctaaatcaacgaa $\mathrm{C} / \mathrm{G}$ tgattcctggagagcagctt & & & rs1340361 & \\
\hline 18 & $23063 \mathrm{G}>\mathrm{A}$ intron 1 & & ggaggcacetgtgacaccca $\mathrm{G} / \mathrm{A}$ cggagtaggggggcggtgtg & & & & \\
\hline 19 & $23131 \mathrm{G}>\mathrm{A}$ intron 1 & & agtgtgcatatgtgctgacc $\mathrm{G} / \mathrm{A}$ tgggagcttgtttgtcggtt & & & & \\
\hline 20 & $156 \mathrm{~T}>\mathrm{C}$ intron 2 & & ggacacaggactgtgtggtc $\mathrm{T} / \mathrm{C}$ ggatatggcatgtggcttat & & & rs1078143 & \\
\hline 21 & $384 \mathrm{~A}>\mathrm{G}$ intron 2 & & gctgtgggtgaagtgagtta $\mathrm{A} / \mathrm{G}$ tggccccactcttagagatc & & & rs1078144 & \\
\hline 22 & $1081 \mathrm{G}>\mathrm{A}$ intron 2 & & agtgcagccaaaattgcaaa G/A tcataccattcaaattaata & & & rs752187 & \\
\hline 23 & $2801 \mathrm{~A}>\mathrm{G}$ intron 2 & & aagaaaagtgatttatttca $\mathrm{A} / \mathrm{G}$ gttgctgatgcttagattgt & & & & \\
\hline 24 & $2830 \mathrm{C}>\mathrm{G}$ intron 2 & & tgcttagattgttagagttg $\mathrm{C} / \mathrm{G}$ aaagatctggettgcatctt & & & & \\
\hline 25 & $2856 \mathrm{~A}>\mathrm{G}$ intron 2 & & tctggcttgcatcttgtaca $\mathrm{A} / \mathrm{G}$ ctgacagaactggggctcag & & & & \\
\hline 26 & $3187 \mathrm{~A}>\mathrm{G}$ intron 2 & & tgatagctgttgcctgcagc $\mathrm{A} / \mathrm{G}$ tacggacgttcattgcgcag & & & & \\
\hline 27 & $3190 \mathrm{C}>\mathrm{T}$ intron 2 & & tagctgttgectgcagcata $\mathrm{C} / \mathrm{T}$ ggacgttcattgcgcagttc & & & & \\
\hline 28 & $3194 \mathrm{C}>\mathrm{T}$ intron 2 & & tgttgcctgcagcatacgga $C / T$ gttcattgcgcagttcetgt & & & & \\
\hline 29 & $3204 \mathrm{G}>\mathrm{A}$ intron 2 & & agcatacggacgttcattgc $\mathrm{G} / \mathrm{A}$ cagttcctgtctcctgagat & & & & \\
\hline 30 & $3401 \mathrm{~T}>\mathrm{C}$ intron 2 & & acataaagcctgtgtgctgc $\mathrm{T} / \mathrm{C}$ gccaggaagactagaaacgc & & & & \\
\hline 31 & $13927 \mathrm{~A}>\mathrm{G}$ intron 2 & & gtcaccacatacetggcact $\mathrm{A} / \mathrm{G}$ tgctaaggctgggaatgcag & & $\mathrm{L} 2$ & & \\
\hline 32 & $4163 \mathrm{G}>\mathrm{A}$ intron 3 & & ccagcccacttcatcttacc G/A tagttacctccttagagtat & & & & \\
\hline 33 & $4262 \mathrm{~T}>\mathrm{C}$ intron 3 & & tgtcaaagaggaactaagga $\mathrm{T} / \mathrm{C}$ gccagggactttctgcttag & & & & \\
\hline 34 & $4306 \mathrm{C}>\mathrm{T}$ intron 3 & & ccctctcatcacttctccaa $\mathrm{C} / \mathrm{T}$ gctggtatcatgaaccccat & & & & \\
\hline 35 & $240 \mathrm{G}>\mathrm{A}$ intron 5 & & gacagaagaaaagtccccag G/A gaagaatactacagacttgg & & & rs1107281 & \\
\hline 36 & $490 \mathrm{G}>\mathrm{A}$ intron 5 & & gatgggcatttgaacttgtt $\mathrm{G} / \mathrm{A}$ tctttaaaaagtgaaatctt & & & & \\
\hline 37 & $583 \mathrm{~T}>\mathrm{G}$ intron 5 & & tatctggggagtgggcattt $\mathrm{T} / \mathrm{G}$ ctgactgaggcattggctgc & & & & \\
\hline 38 & $1051 \mathrm{C}>\mathrm{T}$ inton 5 & & ggctacaaaactgtgctttc $\mathrm{C} / \mathrm{T}$ ttgggcagtaaaagaggcaa & & & & \\
\hline 39 & $3051 \mathrm{G}>\mathrm{A}$ intron 5 & & tagagaacaagtctaattct $\mathrm{G} / \mathrm{A}$ ttttccttgaaatagtcgaa & & & & \\
\hline 40 & $3127 \mathrm{~A}>\mathrm{G}$ intron 5 & & aagtccatgattttttaggc $\mathrm{A} / \mathrm{G}$ aaatggectcctttcctctt & & & & \\
\hline 41 & $5924 \mathrm{C}>\mathrm{T}$ intron 5 & & ctttctttcacaaaattgcc $\mathrm{C} / \mathrm{T}$ cccagagctttctggaaggg & & & & \\
\hline 42 & $6831 \mathrm{~T}>\mathrm{C}$ intron 5 & & ccagtccctcagccttgcca $\mathrm{T} / \mathrm{C}$ tgcttatgctggtctggaaa & & & & \\
\hline 43 & $12678 \mathrm{G}>\mathrm{C}$ intron 5 & & gctcaccgctctgctcacce $\mathrm{G} / \mathrm{C}$ accetctggecatctcctct & & & & \\
\hline 44 & $14214 \mathrm{G}>\mathrm{A}$ intron 5 & & cagcttggtcccagaggcct $\mathrm{G} / \mathrm{A}$ gacctgggtcccagaggtcc & & & & \\
\hline 45 & $14257 \mathrm{C}>\mathrm{T}$ intron 5 & & gctggttccecggettggtc $\mathrm{C} / \mathrm{T}$ cagaggcctggatgtgtggc & & & & \\
\hline 46 & $18078 \mathrm{C}>\mathrm{T}$ intron 5 & & cctaccacaccatgcacgtg $\mathrm{C} / \mathrm{T}$ acagccaagggttgttgact & & & & \\
\hline 47 & $18795 \mathrm{G}>\mathrm{A}$ intron 5 & & ctgggctcttcctggacctg G/A ccagctaaaaggaaatctcc & & & & \\
\hline 48 & $18948 \mathrm{G}>\mathrm{A}$ intron 5 & & gcattggtggtactaagaac $\mathrm{G} / \mathrm{A}$ catattccetatcctatagg & & $\mathrm{L} 2$ & & \\
\hline 49 & $19053 \mathrm{~T}>\mathrm{C}$ intron 5 & & ctcccccaacattaaaagtg $\mathrm{T} / \mathrm{C}$ aagggatgcttattcaaatg & & MER5A & & \\
\hline 50 & $19148 \mathrm{C}>\mathrm{A}$ intron 5 & & ggcccaagaaactgcatttt C/A gcatgctccetaaatgaagc & & MER5A & & \\
\hline 51 & $19229 \mathrm{C}>\mathrm{T}$ intron 5 & & atgctaacagtgtagagtca $\mathrm{C} / \mathrm{T}$ atgtgatgggaagcatcagg & & & & \\
\hline 52 & $19405 \mathrm{~T}>\mathrm{C}$ intron 5 & & cttgctcaatttattctgtc $\mathrm{T} / \mathrm{C}$ atataactcaatattactga & & & & \\
\hline 53 & $19534 \mathrm{G}>\mathrm{A}$ intron 5 & & catgtgaccctcttagctcc $\mathrm{G} / \mathrm{A}$ cggattaactcctgtcctca & & & & \\
\hline 54 & $474 \mathrm{G}>\mathrm{A}$ coding region & 6 & gaaaccttctctgggttcct $\mathrm{G} / \mathrm{A}$ tatcacaacctctctctccc & Leu 158 Leu & & & \\
\hline 55 & $210 \mathrm{~A}>\mathrm{C}$ intron 6 & & gcaacctggcgtcatgggcc $\mathrm{A} / \mathrm{C}$ gctggttaaaataaaattga & & & & \\
\hline 56 & $334 \mathrm{G}>\mathrm{A}$ intron 6 & & acagttctgaggcaataacc $\mathrm{G} / \mathrm{A}$ tggttaagggttattgatct & & & & \\
\hline 57 & $2288 \mathrm{C}>\mathrm{T}$ intron 6 & & cttctttcaaagcttgtggt $\mathrm{C} / \mathrm{T}$ cactggaccacgtatgaagt & & & & \\
\hline 58 & $2322 \mathrm{~T}>\mathrm{C}$ intron 6 & & atgaagtagaatagtttagg $\mathrm{T} / \mathrm{C}$ ccagaaaggcaattaagtaa & & & & \\
\hline 59 & $2820 \mathrm{~T}>\mathrm{G}$ intron 6 & & gtgctttgatacattctgag $\mathrm{T} / \mathrm{G}$ ttcagtaaagagacctgatg & & & & \\
\hline 60 & $656 \mathrm{G}>$ A coding region & 7 & tgagctttgtggcctaccaa G/A ggagaaactggctgcagcag & Arg 219 Lys & & & Clee et al. 2001 \\
\hline 61 & $416 \mathrm{G}>\mathrm{A}$ intron 7 & & catcataaagatgacattgt G/A ggctgtcacagttggaaggc & & & & \\
\hline 62 & $471 \mathrm{C}>\mathrm{T}$ intron 7 & & agaccacactatttagctta $\mathrm{C} / \mathrm{T}$ ttagtaataacattgcaaag & & & & \\
\hline 63 & $504 \mathrm{G}>\mathrm{A}$ intron 7 & & ttgcaaagaaaaattccgac G/A aagtttttcagcctaggaa & & & & \\
\hline 64 & $679 \mathrm{G}>\mathrm{C}$ intron 7 & & gctctggtgaaattcctctc $\mathrm{G} / \mathrm{C}$ ctaccccaaacatcatcatt & & & & \\
\hline 65 & $1740 \mathrm{C}>\mathrm{T}$ intron 7 & & acaaatgctcaccctttcag $\mathrm{C} / \mathrm{T}$ tggaatgattgaaattttgg & & & & \\
\hline 66 & $2122 \mathrm{~A}>\mathrm{G}$ intron 7 & & tgattaaggtggctactacc $\mathrm{A} / \mathrm{G}$ ggtgctttctgcatatctcg & & & & \\
\hline 67 & $7753 \mathrm{~T}>\mathrm{C}$ intrion 7 & & taggaattccaagctgtgaa $\mathrm{T} / \mathrm{C}$ tttttactgaagctctttgg & & & & \\
\hline 68 & $8973 \mathrm{~A}>\mathrm{T}$ intron 7 & & atggaaatttgtttatattg $\mathrm{A} / \mathrm{T}$ ctacagattgccaatattat & & & & \\
\hline 69 & $8976 \mathrm{~A}>\mathrm{G}$ intron 7 & & gaaatttgtttatattgact $\mathrm{A} / \mathrm{G}$ cagattgccaatattattag & & & & \\
\hline
\end{tabular}


Table 1. Continued

\begin{tabular}{|c|c|c|c|c|c|c|c|}
\hline Number & Location & Exon & $\operatorname{SNP}\left(5^{\prime}\right.$ to $\left.3^{\prime}\right)$ & Substituion & $\begin{array}{l}\text { Repetitive } \\
\text { sequence }\end{array}$ & $\begin{array}{l}\text { Identity } \\
\text { to dbSNP }\end{array}$ & Reference \\
\hline 70 & $11327 \mathrm{G}>\mathrm{C}$ intron 7 & & ctaacaatcttatttccatt $\mathrm{G} / \mathrm{C}$ agtccttataaaagaagtgg & & & & \\
\hline 71 & $11738 \mathrm{C}>\mathrm{T}$ intron 7 & & ctgacgtttaagggagaccg $\mathrm{C} / \mathrm{T}$ gtaggtccetttgaggactg & & & & \\
\hline 72 & $12295 \mathrm{~T}>\mathrm{A}$ intron 7 & & agtctgtaaattattgttct $\mathrm{T} / \mathrm{A}$ tttttctttagcttatgct & & & & \\
\hline 73 & $387 \mathrm{C}>\mathrm{G}$ intron 8 & & tagcaaggccaatcatttta $\mathrm{C} / \mathrm{G}$ caacacacatgcttgctaac & & & & \\
\hline 74 & $697 \mathrm{~A}>\mathrm{T}$ intron 8 & & ggaactgtctggtgtccccc $\mathrm{A} / \mathrm{T}$ gcataggaagctgagccagg & & & & \\
\hline 75 & $1312 \mathrm{G}>\mathrm{A}$ intron 8 & & attgctctgcagatcccctc $\mathrm{G} / \mathrm{A}$ cagccctetgtccettgttc & & & rs1175929 & \\
\hline 76 & $3036 \mathrm{~T}>\mathrm{G}$ intron 8 & & ctttatgtgggaagaaattt T/G ttttttgattggggagtgg & & & & \\
\hline 77 & $3176 \mathrm{C}>\mathrm{A}$ intron 8 & & aaatggcctggttctctgtc $\mathrm{C} / \mathrm{A}$ cctttctgtctgtatgectc & & & & \\
\hline 78 & $3364 \mathrm{~A}>\mathrm{T}$ intron 8 & & ggcagaaggcaaagcttagg $\mathrm{A} / \mathrm{T}$ cctagagagtgctggaccac & & & & \\
\hline 79 & $3373 \mathrm{G}>\mathrm{A}$ intron 8 & & caaagcttaggacctagaga G/A tgctggaccacgccactcac & & & & \\
\hline 80 & $3561 \mathrm{C}>\mathrm{A}$ intron 8 & & cagggatttattaatgattt $\mathrm{C} / \mathrm{A}$ ttgtgaaatgtttggaaata & & & & \\
\hline 81 & $3654 \mathrm{~T}>\mathrm{C}$ intron 8 & & agtgccggaatacatttgca $T / C$ gtaagacagaacgctgcctg & & & & \\
\hline 82 & $4715 \mathrm{C}>\mathrm{T}$ intron 8 & & ggcagaggggtctcagaatc $\mathrm{C} / \mathrm{T}$ gcatttccaacaatgtctcc & & & & \\
\hline 83 & $936 \mathrm{C}>\mathrm{T}$ coding region & 9 & cgtattgtctgcgggcatcc $\mathrm{C} / \mathrm{T}$ gagggaggggggctgaagat & Pro 312 Pro & & & \\
\hline 84 & $2309 \mathrm{~A}>\mathrm{G}$ intron 9 & & cccctcaagagtcagtttaa $\mathrm{A} / \mathrm{G}$ tgttggtcatgttagttgtc & & & & \\
\hline 85 & $2392 \mathrm{~T}>\mathrm{C}$ intron 9 & & atgggagggcttgtgcttca $\mathrm{T} / \mathrm{C}$ gaaaacatttttccagatca & & & & \\
\hline 86 & $228 \mathrm{~A}>\mathrm{G}$ intron 10 & & tggggatggggaggactgge $\mathrm{A} / \mathrm{G}$ cagggctgctgtgatggggt & & & & \\
\hline 87 & $319 \mathrm{C}>\mathrm{T}$ intron 10 & & ttctgcggtccctggctccc $\mathrm{C} / \mathrm{T}$ acctgactccaggtgaacaa & & & & \\
\hline 88 & $377 \mathrm{~A}>\mathrm{C}$ intron 11 & & gaaagaagtgtgggagcaaa $\mathrm{A} / \mathrm{C}$ gcatgatgttacatgtagac & & & & \\
\hline 89 & $521 \mathrm{G}>\mathrm{A}$ intron 11 & & agtgctctagagacaattgg G/A ttcaaatgtggagcaggctg & & & & \\
\hline 90 & $2850 \mathrm{G}>\mathrm{C}$ intron 11 & & ctctatacaatcattatgct $\mathrm{G} / \mathrm{C}$ ccattgaaataataaataca & & & & \\
\hline 91 & $2976 \mathrm{~A}>\mathrm{G}$ intron 11 & & ctccaattcggtagaaccag $\mathrm{A} / \mathrm{G}$ gcttcatcttctctgtcgaa & & & & \\
\hline 92 & $3056 \mathrm{C}>\mathrm{T}$ intron 11 & & gtttgcagctgctgtttttc $\mathrm{C} / \mathrm{T}$ ggcagcacatctgtgcaggc & & & & \\
\hline 93 & $340 \mathrm{~T}>\mathrm{C}$ intron 12 & & ggcattatttgtgaaactta $\mathrm{T} / \mathrm{C}$ ctaaaatcgaattcgggtcc & & & & \\
\hline 94 & $381 \mathrm{~A}>\mathrm{G}$ intron 12 & & aattaaattttgaaatttt $\mathrm{A} / \mathrm{G}$ tattaaaaattatattagta & & & & \\
\hline 95 & $1728 \mathrm{C}>\mathrm{T}$ intron 14 & & caggctcagaggccttggcc $\mathrm{C} / \mathrm{T}$ atcaccctggctcacgtgtg & & & & \\
\hline 96 & $2040 \mathrm{C}>\mathrm{A}$ coding region & 15 & atgggcctggacaacagcat $\mathrm{C} / \mathrm{A}$ ctctggtttagctggttcat & Ile 680 Ile & & & \\
\hline 97 & $1382 \mathrm{G}>\mathrm{A}$ intron 15 & & cttttagacagaaaagttac $\mathrm{G} / \mathrm{A}$ tgggatattatctcccacag & & & & \\
\hline 98 & $1453 \mathrm{G}>\mathrm{A}$ intron 15 & & tatataaggagaaaccagtt G/A aaattacctattgaagaaac & & & & \\
\hline 99 & $1567 \mathrm{G}>\mathrm{A}$ intron 15 & & ttctgcgtagttttgggtaa $\mathrm{G} / \mathrm{A}$ tcacttatcttctttaggat & & MIR & & \\
\hline 100 & $1617 \mathrm{~T}>\mathrm{A}$ intron 15 & & cagttgcctcatcagaaaga T/A gaacagcattacgcctctgc & & MIR & & \\
\hline 101 & $95 \mathrm{~T}>\mathrm{A}$ intron 16 & & agttgagaacagaagatgat $\mathrm{T} / \mathrm{A}$ gtcttttccaatgggacatg & & & & \\
\hline 102 & $452 \mathrm{G}>\mathrm{A}$ intron 16 & & tggtgttttgettgagtaat $\mathrm{G} / \mathrm{A}$ ttttctgaactaagcacaac & & & & \\
\hline 103 & $657 \mathrm{~T}>\mathrm{C}$ intron 16 & & ctgttgcctcagtctgggct $\mathrm{T} / \mathrm{C}$ cataggcatcagcagcccca & & & & \\
\hline 104 & $2473 \mathrm{G}>\mathrm{A}$ coding region & 17 & gcttcaatctcaccacttcg G/A tctccatgatgctgtttgac & Val 825 Ile & & & Clee et al. 2001 \\
\hline 105 & $2649 \mathrm{~A}>\mathrm{G}$ coding region & 18 & ggttccaaccagaagagaat $\mathrm{A} / \mathrm{G}$ tcagaaagtaagtgctgttg & Ile $883 \mathrm{Met}$ & & & Clee et al. 2001 \\
\hline 106 & $1730 \mathrm{C}>\mathrm{G}$ intron 18 & & tgaaagttcaagcgcagtgc $\mathrm{C} / \mathrm{G}$ ctgtgtcettacactccact & & & & \\
\hline 107 & $426 \mathrm{~A}>\mathrm{G}$ intron 19 & & aggaccttacagtgggtagt $\mathrm{A} / \mathrm{G}$ tcaggaggggtcaggggctg & & & & \\
\hline 108 & $468 \mathrm{~A}>\mathrm{G}$ intron 19 & & aaagcaccagcgttagcctc $\mathrm{A} / \mathrm{G}$ gtggettccagcacgattcc & & & & \\
\hline 109 & $876 \mathrm{C}>\mathrm{T}$ intron 20 & & ccctcctcatctaaagtgaa $\mathrm{C} / \mathrm{T}$ acatggggctcatgtgcagg & & & & \\
\hline 110 & $118 \mathrm{~T}>\mathrm{G}$ intron 22 & & catgggatactcttctgtta T/G cacagaagagataaagggca & & & & \\
\hline 111 & $560 \mathrm{G}>\mathrm{A}$ intron 22 & & aaagctttgccattctaggg $\mathrm{G} / \mathrm{A}$ tcatagccatacagggtgaa & & & & \\
\hline 112 & $102 \mathrm{~A}>\mathrm{G}$ intron 23 & & accccttttgccatgttgaa $\mathrm{A} / \mathrm{G}$ ccaccatctccetgctctgt & & & & \\
\hline 113 & $287 \mathrm{C}>\mathrm{T}$ intron 23 & & gtcaaagaaaagagacttgt $\mathrm{C} / \mathrm{T}$ aagaggtaagagccttggct & & & & \\
\hline 114 & $1063 \mathrm{G}>\mathrm{A}$ intron 23 & & acetttcaccctcaggaagc G/A aggetgttcacacggcacac & & & & \\
\hline 115 & $321 \mathrm{~T}>\mathrm{G}$ intron 25 & & ctctttacttaagtacagtg T/G gaggaacagcggcatcagga & & MER5A & & \\
\hline 116 & $376 \mathrm{G}>\mathrm{C}$ intron 25 & & gttagaaattcagcaacttg G/C gcccagctcagacctactga & & MER5A & & \\
\hline 117 & $478 \mathrm{C}>\mathrm{T}$ intron 25 & & catacataggaaatgacaaa $\mathrm{C} / \mathrm{T}$ gtttatggatggatagtcta & & & & \\
\hline 118 & $579 \mathrm{G}>\mathrm{T}$ intron 25 & & tcatttaattctcaaaaaaa $\mathrm{G} / \mathrm{T}$ atgaaaaaatgaacactcag & & & & \\
\hline 119 & $153 \mathrm{C}>\mathrm{T}$ intron 27 & & aatggtaaaagccacttgtt $\mathrm{C} / \mathrm{T}$ tttgcagcatcgtgcatgtg & & & & \\
\hline 120 & $1058 \mathrm{C}>\mathrm{T}$ intron 28 & & actatcatgggagataatga $\mathrm{C} / \mathrm{T}$ tatggttgtccatgattgga & & & & \\
\hline 121 & $1317 \mathrm{C}>\mathrm{T}$ intron 28 & & caggacccagtgttctgagt $\mathrm{C} / \mathrm{T}$ accctgaatgtgagcactat & & & & \\
\hline 122 & $372 \mathrm{~T}>\mathrm{C}$ intron 30 & & tatatgatttttaggttttg $\mathrm{T} / \mathrm{C}$ ttatcagcttcttcgcttt & & & & \\
\hline 123 & $506 \mathrm{~A}>\mathrm{G}$ intron 30 & & ccttttaaaaagtaagcagt A/G gataaataaattcagtgaag & & & & \\
\hline 124 & $1033 \mathrm{G}>\mathrm{C}$ intron 30 & & ctggatttcatggtgccttt $\mathrm{G} / \mathrm{C}$ attttccacatgaaggttgt & & & & \\
\hline 125 & $4281 \mathrm{G}>\mathrm{A}$ coding region & 31 & tcttccetttgcagagacac G/A ccctgccaggcaggggagga & Thr $1427 \mathrm{Thr}$ & & & \\
\hline 126 & $626 \mathrm{C}>\mathrm{T}$ intron 33 & & ggctccttgttactgatttc $\mathrm{C} / \mathrm{T}$ gtcttttctctctgectttt & & & & \\
\hline 127 & $719 \mathrm{G}>\mathrm{A}$ intron 33 & & taatagccctcatgctagaa G/A ggagccggagcctgtgtata & & & & \\
\hline 128 & $726 \mathrm{G}>\mathrm{A}$ intron 33 & & cctcatgctagaagggagcc G/A gagcctgtgtataaggccag & & & & \\
\hline 129 & $889 \mathrm{~A}>\mathrm{G}$ intron 33 & & ctttcctcaatgtctcagct $\mathrm{A} / \mathrm{G}$ tctaactgtgtgtgtaatca & & & & \\
\hline 130 & $1097 \mathrm{G}>\mathrm{C}$ intron 33 & & ctgtgcaccccactgtctgg $\mathrm{G} / \mathrm{C}$ ttttaatgtcaggctgttct & & & & \\
\hline 131 & $4760 \mathrm{G}>\mathrm{A}$ coding region & 35 & tatgacaggactggacacca $\mathrm{G} / \mathrm{A}$ aaataatgtcaaggtaaacc & Arg 1587 Lys & & & Clee et al. 2001 \\
\hline 132 & $234 \mathrm{~T}>\mathrm{C}$ intron 35 & & aacctatctaaacctcagtt $\mathrm{T} / \mathrm{C}$ cctcatctgtgaaatggaga & & MIR & & \\
\hline 133 & $411 \mathrm{C}>\mathrm{T}$ intron 37 & & aactctgtacattttatcag $\mathrm{C} / \mathrm{T}$ agcttatccatccattgcaa & & & & \\
\hline 134 & $1224 \mathrm{~A}>\mathrm{G}$ intron 37 & & caggcataggtgattcagag A/G tgaaaggtcaagtccctgaa & & L2 & & \\
\hline 135 & $1720 \mathrm{G}>\mathrm{T}$ intron 37 & & aaattaaaattactctgact $\mathrm{G} / \mathrm{T}$ ggaatccatcgttcagtaag & & & & \\
\hline 136 & $251 \mathrm{~T}>\mathrm{G}$ intron 40 & & tgaaggtaaggaaaatagtg $\mathrm{T} / \mathrm{G}$ tatttgcttggatccactgg & & & & \\
\hline 137 & $252 \mathrm{~T}>\mathrm{C}$ intron 40 & & gaaggtaaggaaaatagtgt $\mathrm{T} / \mathrm{C}$ atttgcttggatccactggc & & & & \\
\hline 138 & $319 \mathrm{~A}>\mathrm{G}$ intron 40 & & agcactggaaaagtcaaacc $\mathrm{A} / \mathrm{G}$ taactttgagaattaggtga & & & & \\
\hline
\end{tabular}


Table 1. Continued

\begin{tabular}{|c|c|c|c|c|c|c|c|}
\hline Number & Location & Exon & $\operatorname{SNP}\left(5^{\prime}\right.$ to $\left.3^{\prime}\right)$ & Substituion & $\begin{array}{l}\text { Repetitive } \\
\text { sequence }\end{array}$ & $\begin{array}{l}\text { Identity } \\
\text { to dbSNP }\end{array}$ & Reference \\
\hline 139 & $957 \mathrm{G}>\mathrm{C}$ intron 40 & & cttgttactctttttcctt $\mathrm{G} / \mathrm{C}$ tcatgggtgatagccatttg & & & & \\
\hline 140 & $146 \mathrm{C}>\mathrm{T}$ intron 41 & & tgatgtgggcatcccgcagc $\mathrm{C} / \mathrm{T}$ ccetccetgcccatcctgga & & & & \\
\hline 141 & $239 \mathrm{~A}>\mathrm{C}$ intron 42 & & cattggtttatatgcttac $\mathrm{A} / \mathrm{C}$ tttatgtgttagttattaaa & & & & \\
\hline 142 & $321 \mathrm{~T}>\mathrm{A}$ intron 42 & & aataaatggttgattttgag $\mathrm{T} / \mathrm{A}$ ttgagtttcatagtccaaaa & & & & \\
\hline 143 & $322 \mathrm{~T}>\mathrm{C}$ intron 42 & & ataaatggttgattttgagt $\mathrm{T} / \mathrm{C}$ tgagtttcatagtccaaaaa & & & & \\
\hline 144 & $533 \mathrm{G}>\mathrm{A}$ intron 42 & & agatgaaaaattatgtagat $\mathrm{G} / \mathrm{A}$ ataatgaatgatacggttct & & & & \\
\hline 145 & $546 \mathrm{~A}>\mathrm{G}$ intron 42 & & tgtagatgataatgaatgat $\mathrm{A} / \mathrm{G}$ cggttctaaaaagacaggtt & & & & \\
\hline 146 & $739 \mathrm{~T}>\mathrm{A}$ intron 43 & & tacagccacacttaaaatgg T/A cccattatgaaatacatatt & & & & \\
\hline 147 & $18 \mathrm{~T}>\mathrm{C}$ intron 44 & & taggtgagaaaagaagtggc $\mathrm{T} / \mathrm{C}$ tgtattttgctgcaaagact & & & & \\
\hline 148 & $264 \mathrm{~T}>\mathrm{C}$ intron 44 & & acaatataatttgcttgttt $\mathrm{T} / \mathrm{C}$ ttaagagtataatttagtga & & L1MB8 & & \\
\hline 149 & $279 \mathrm{~T}>\mathrm{C}$ intron 44 & & tgtttttaagagtataatt $\mathrm{T} / \mathrm{C}$ agtgattttggtaaattga & & L1MB8 & & \\
\hline 150 & $508 \mathrm{C}>\mathrm{T}$ intron 44 & & tttacattgctacataaaat $\mathrm{C} / \mathrm{T}$ cccctatgtacatgtaccta & & & & \\
\hline 151 & $1477 \mathrm{~A}>\mathrm{T}$ intron 44 & & gatctcctctcctgtctctt $\mathrm{A} / \mathrm{T}$ cattttgcagtagcaatgt & & & & \\
\hline 152 & $1665 \mathrm{G}>\mathrm{A}$ intron 44 & & tggttgtaagaactgatttg G/A ttggtatagctgtgagggcc & & & & \\
\hline 153 & $1956 \mathrm{~T}>\mathrm{G}$ intron 44 & & gtgttgctcacactcaaaat $\mathrm{T} / \mathrm{G}$ tctgggccttctcatttggt & & & & \\
\hline 154 & $68 \mathrm{~T}>\mathrm{C}$ intron 45 & & aatatataccttatggcttt $\mathrm{T} / \mathrm{C}$ ccacacgcattgacttcagg & & & & \\
\hline 155 & $608 \mathrm{G}>\mathrm{C}$ intron 46 & & ttatactgacttcaatagag G/C tttcagacaaaaagttgttt & & & & \\
\hline 156 & $336 \mathrm{~T}>\mathrm{C}$ intron 47 & & ttcacaattgtaaacaccac $\mathrm{T} / \mathrm{C}$ acactgaacagcatcatccc & & L1MD2 & & \\
\hline 157 & $55 \mathrm{G}>\mathrm{C}$ intron 49 & & agggtgtggattcctgcccc $\mathrm{G} / \mathrm{C}$ acactcccgcccataggtcc & & & rs1331924 & \\
\hline 158 & $7479 \mathrm{C}>\mathrm{T} 3^{\prime}$ untranslated region & 50 & aacaaaaatgtgggtgtctc $\mathrm{C} / \mathrm{T}$ aggcacgggaaacttggttc & & & & \\
\hline 159 & $8226 \mathrm{C}>\mathrm{T} 3^{\prime}$ untranslated region & 50 & aggagcccactgtaacaata $\mathrm{C} / \mathrm{T}$ tgggcagcctttttttttt & & & & \\
\hline 160 & $8682 \mathrm{G}>\mathrm{A} 3^{\prime}$ untranslated region & 50 & aacttcttccactttttcca G/A aatttgaatattaacgctaa & & & rs 363717 & \\
\hline 161 & $8697 \mathrm{C}>\mathrm{T}$ 3' untranslated region & 50 & ttccagaatttgaatattaa $\mathrm{C} / \mathrm{T}$ gctaaaggtgtaagacttca & & & & \\
\hline 162 & $9097 \mathrm{~A}>$ G 3' untranslated region & 50 & aactattttgaagaaaacac $\mathrm{A} / \mathrm{G}$ acattttaatacagattgaa & & & & \\
\hline
\end{tabular}

Nucleotide numbering is according to the mutation nomenclature (den Dunnen and Antonarakis, 2000)

Table 2. Characterization of insertion/deletion polymorphisms at the $A B C A 1$ locus

\begin{tabular}{|c|c|c|}
\hline Number & Location & Variations $\left(5^{\prime}\right.$ to $\left.3^{\prime}\right)$ \\
\hline 1 & $(-1033)-(-1032)$ ins AT 5' flanking region & tgacttaaatatttagacat $(\mathrm{AT} /+)$ ggtgtgtaggcctgcattcc \\
\hline 2 & 6368 del C intron 5 & ttctgatggggttgttgctg $(\mathrm{C} /-)$ tgagaatcatgactgggtgg \\
\hline 3 & 9709 del $\mathrm{T}$ intron 5 & cattttctgtctgaaccccc $(\mathrm{T} /-)$ cacccattcaggcagctgct \\
\hline 4 & 13816 del $\mathrm{T}$ intron 5 & tccetacttctccttttttt $(\mathrm{T} /-)$ catttgcetcctccacccac \\
\hline 5 & $270-271$ ins $\mathrm{G}$ intron 10 & cttttcagggaggagccaaa $(\mathrm{G} /+)$ cgctcattgtctgtgcttct \\
\hline 6 & $611-612$ ins $C$ intron 20 & tttagcccatcctctccec $(\mathrm{C} /+)$ gccaccetccttattgaggc \\
\hline 7 & 391-392 ins $\mathrm{T}$ intron 32 & gagtgccttgggtactctct $(T /+)$ gatgggggactccatgataa \\
\hline 8 & 847 del C intron 37 & gctgtatattgtgaatgtcc $(\mathrm{C} /-)$ gttttcaaaagcaaagccaa \\
\hline
\end{tabular}

Nucleotide numbering is according to the mutation nomenclature (den Dunnen and Antonarakis, 2000) $(+)$, insertion polymorphism; $(-)$, deletion polymorphism

is an important resource for understanding not only the etiology and risk of some diseases, but also the pharmacokinetics or pharmacodyamics of drugs used to treat them. We have focused on identifying variations in genomic regions that contain drug-metabolizing enzymes, and so far have isolated 1066 SNPs among 71 such loci (lida et al. 2001a,b; Saito et al. 2001; Saito et al., in preparation; Sekine et al. 2001). We report here a total of 162 SNPs spanning the entire $A B C A 1$ genomic region, detected among 96 chromosomes in a Japanese population sample.

\section{Subjects and methods}

DNA samples, PCR, direct sequencing, and detection of SNPs

Blood samples were obtained with informed consent from 48 healthy Japanese individuals for this study, which was approved by the ethical committee of the RIKEN SNP Research Center. On the basis of $A B C A 1$-genomic sequences (accession numbers AF275948.1 and AL359846.11), released from the GenBank database, and information published by Remaley et al. (1999) and Santamarina-Fojo et al. (2000), we designed primers to amplify the $A B C A 1$ gene in its entirety as well as up to $2 \mathrm{~kb}$ upstream from the first exon and downstream of the last exon. We eliminated most of the regions corresponding to repetitive sequences predicted by the RepeatMasker program (http://ftp. genome.washington.edu/cgibin/ RepeatMasker). Genomic DNA extraction, polymerase chain reaction (PCR) experiments, and DNA sequencing were performed according to methods described previously (Ohnishi et al. 2000). Each PCR was performed using 20ng of DNA pooled from three individuals. All SNPs detected by the PolyPhred computer program (Nickerson et al. 1997) were confirmed by sequencing both strands of each PCR product. 


\section{Results and discussion}

We present here a highly dense SNP map covering the $150-\mathrm{kb}$ genomic region that contains the entire human $A B C A 1$ gene. A total of 91 partially overlapping PCR fragments, covering $57.4 \%$ of the $150-\mathrm{kb} A B C A 1$ gene locus, were sequenced using 364 primers. The organization of the $A B C A 1$ gene and locations of identified SNPs are illustrated schematically in Fig. 1. A total of 162 SNPs were identified: eight were located in coding elements, two in the $5^{\prime}$ flanking region, 147 in introns, and five in the $3^{\prime}$ untranslated region (Table 1; also see Fig. 1). No SNP was detected in the 5' untranslated or 3' flanking regions. Comparison of our data with SNPs deposited in the dbSNP database in the National Center for Biotechnology Information (NCBI, U.S.) and an earlier report (Clee et al. 2001) indicated that 149 (94\%) of the SNPs identified in this study could be considered novel. As a whole, 154 of the 162 SNPs identified (95.0\%) were located within $79.4 \mathrm{~kb}$ of DNA representing noncoding exons, introns, and resequenced flanking regions (1SNP/ $516 \mathrm{bp}$ ), whereas only 8 SNPs were present in $6783 \mathrm{bp}$ of the coding DNA screened (1SNP/848bp). Therefore, the frequency of SNPs in noncoding portions of the $A B C A 1$ gene was 1.64-fold higher than in coding elements. Frequencies of the substitutions were $35.8 \%$ for $\mathrm{A} / \mathrm{G}, 34.6 \%$ for $\mathrm{C} / \mathrm{T}, 11.7 \%$ for $\mathrm{C} / \mathrm{G}, 6.8 \%$ for $\mathrm{G} / \mathrm{T}, 6.2 \%$ for $\mathrm{A} / \mathrm{T}$, and $4.9 \%$ for $\mathrm{A} / \mathrm{C}$. The ratio of transitions to transversions was approximately 2.37 to 1 . The higher prevalence of $\mathrm{A} / \mathrm{G}$ substitutions and the transition/transversion ratio are both in close accord with previous observations (Cambien et al. 1999; Iida et al. 2001a,b; Venter et al. 2001). In addition to SNPs, we identified eight novel insertion/deletion polymorphisms within introns of the $A B C A 1$ gene (Table 2).

Among 13 SNPs identified in exonic regions, the 4 nonsynonymous sites that we found in coding elements had been reported previously (Clee et al. 2001). Although the frequency of the coding SNPs, particularly nonsynonymous ones, was very small, they would be likely to influence protein function. In fact, it is reported that the $\mathrm{R} 219 \mathrm{~K}$ substitution is associated with a decreased severity of atherosclerosis, a decreased risk of coronary events, decreased triglycerides, and a higher level of HDL cholesterol (HDLC) in plasma (Clee et al. 2001). Moreover, since the regulatory elements for gene expression are contained in $5^{\prime}$ promoter regions and introns and occasionally in $3^{\prime}$ flanking regions, SNPs within those regions may affect the quantity of the gene product. In light of recent progress in understanding the biomedical features of $A B C A 1$, the SNPs documented here will be useful not only for studying associations between specific SNPs and some coronary diseases, but also for determining the applicability of pharmacogenomic information to medical practice.

\section{References}

Bodzioch M, Orso E, Klucken J, Langmann T, Bottcher A, Diederich W, Drobnik W, Barlage S, Buchler C, Porsch-Ozcurumez M,
Kaminski WE, Hahmann HW, Oette K, Rothe G, Aslanidis C, Lackner KJ, Schmitz G (1999) The gene encoding ATP-binding cassette transporter 1 is mutated in Tangier disease. Nat Genet 22:347-351

Brooks-Wilson A, Marcil M, Clee SM, Zhang LH, Roomp K, van Dam M, Yu L, Brewer C, Collins JA, Molhuizen HO, Loubser O, Ouelette BF, Fichter K, Ashbourne-Excoffon KJ, Sensen CW, Scherer S, Mott S, Denis M, Martindale D, Frohlich J, Morgan K, Koop B, Pimstone S, Kastelein JJ, Genest J Jr, Hayden MR (1999) Mutations in $A B C 1$ in Tangier disease and familial high-density lipoprotein deficiency. Nat Genet 22:336-345

Cambien F, Poirier O, Nicaud V, Herrmann SM, Mallet C, Ricard S, Behague I, Hallet V, Blanc H, Loukaci V, Thillet J, Evans A, Ruidavets JB, Arveiler D, Luc G, Tiret L (1999) Sequence diversity in 36 candidate genes for cardiovascular disorders. Am J Hum Genet 65:183-191

Clee SM, Zwinderman AH, Engert JC, Zwarts KY, Molhuizen HO, Roomp K, Jukema JW, van Wijland M, van Dam M, Hudson TJ, Brooks-Wilson A, Genest J, Jr, Kastelein JJ, Hayden MR (2001) Common genetic variation in abcal is associated with altered lipoprotein levels and a modified risk for coronary artery disease. Circulation 103:1198-1205

den Dunnen JT, Antonarakis SE (2000) Mutation nomenclature extensions and suggestions to describe complex mutations: a discussion. Hum Mut 15:7-12

Hamon Y, Luciani MF, Becq F, Verrier B, Rubartelli A, Chimini G (1997) Interleukin-1beta secretion is impaired by inhibitors of the Atp binding cassette transporter, ABC1. Blood 90:2911-2915

Hamon Y, Broccardo C, Chambenoit O, Luciani MF, Toti F, Chaslin S, Freyssinet JM, Devaux PF, McNeish J, Marguet D, Chimini G (2000) $A B C 1$ promotes engulfment of apoptotic cells and transbilayer redistribution of phosphatidylserine. Nat Cell Biol 2:399-406

Iida A, Sekine A, Saito A, Kitamura Y, Kitamoto T, Osawa S, Mishima C, Nakamura Y (2001a) Catalog of 320 single nucleotide polymorphisms (SNPs) in 20 quinone oxidoreductase and sulfotransferase genes. J Hum Genet 46:225-240

Iida A, Saito S, Sekine A, Kitamoto T, Kitamura Y, Mishima C, Osawa S, Kondo K, Harigae S, Nakamura Y (2001b) Catalog of 434 single nucleotide polymophisms (SNPs) in the alcohol dehydrogenase genes, glutathione $\mathrm{S}$ transferase genes, and NADH ubiquinone oxidoreductase genes. J Hum Genet 46:385-407

Langmann T, Klucken J, Reil M, Liebisch G, Luciani MF, Chimini G, Kaminski WE, Schmitz G (1999) Molecular cloning of the human ATP-binding cassette transporter $1(h A B C 1)$ : evidence for steroldependent regulation in macrophages. Biochem Biophys Res Commun 1999 257:29-33

Luciani MF, Chimini G (1996) The ATP binding cassette transporter $A B C 1$, is required for the engulfment of corpses generated by apoptotic cell death. EMBO J 15:226-235

Luciani MF, Denizot F, Savary S, Mattei MG, Chimini G (1994) Cloning of two novel ABC transporters mapping on human chromosome 9. Genomics 21:150-159

Marcil M, Brooks-Wilson A, Clee SM, Roomp K, Zhang LH, Yu L, Collins JA, van Dam M, Molhuizen HO, Loubster O, Ouellette BF, Sensen CW, Fichter K, Mott S, Denis M, Boucher B, Pimstone S, Genest J, Jr, Kastelein JJ, Hayden MR (1999) Mutations in the $A B C 1$ gene in familial HDL deficiency with defective cholesterol efflux. Lancet 354:1341-1346

Nickerson DA, Tobe VO, Taylor SL (1997) PolyPhred: automating the detection and genotyping of single nucleotide substitutions using fluorescence-based resequencing. Nucleic Acids Res 25:2745-2751

Ohnishi Y, Tanaka T, Yamada R, Suematsu K, Minami M, Fujii K, Hoki N, Kodama K, Nagata S, Hayashi T, Kinoshita N, Sato H, Sato H, Kuzuya T, Takeda H, Hori M, Nakamura Y (2000) Identification of 187 single nucleotide polymorphisms (SNPs) among 41 candidate genes for ischemic heart disease in the Japanese population. Hum Genet 106:288-292

Oram JF (2000) Tangier disease and ABCA1. Biochim Biophys Acta 1529:321-330

Orso E, Broccardo C, Kaminski WE, Bottcher A, Liebisch G, Drobnik W, Gotz A, Chambenoit O, Diederich W, Langmann T, Spruss T, Luciani MF, Rothe G, Lackner KJ, Chimini G, Schmitz G (2000) Transport of lipids from golgi to plasma membrane is defective in tangier disease patients and $A b c 1$-deficient mice. Nat Genet 24:192196 
Remaley AT, Rust S, Rosier M, Knapper C, Naudin L, Broccardo C, Peterson KM, Koch C, Arnould I, Prades C, Duverger N, Funke H, Assman G, Dinger M, Dean M, Chimini G, Santamarina-Fojo S, Fredrickson DS, Denefle P, Brewer HB, Jr (1999) Human ATPbinding cassette transporter $1(A B C 1)$ : genomic organization and identification of the genetic defect in the original Tangier disease kindred. Proc Natl Acad Sci USA 96:12685-12690

Rust S, Rosier M, Funke H, Real J, Amoura Z, Piette JC, Deleuze JF, Brewer HB, Duverger N, Denefle P, Assmann G (1999) Tangier disease is caused by mutations in the gene encoding ATP-binding cassette transporter 1 . Nat Genet 22:352-355

Saito S, Iida A, Sekine A, Eguchi C, Miura Y, Nakamura Y (2001) Seventy genetic variations in human microsomal and soluble epoxide hydrolase (EPHX1 and $E P H X 2)$ genes in the Japanese population. J Hum Genet 46:325-329
Santamarina-Fojo S, Peterson K, Knapper C, Qiu Y, Freeman L, Cheng JF, Osorio J, Remaley A, Yang XP, Haudenschild C, Prades C, Chimini G, Blackmon E, Francois T, Duverger N, Rubin EM, Rosier M, Denefle P, Fredrickson DS, Brewer HB, Jr (2000) Complete genomic sequence of the human $A B C A 1$ gene: analysis of the human and mouse ATP-binding cassette A promoter. Proc Natl Acad Sci USA 97:7987-7992

Sekine A, Saito S, Iida A, Mitsunobu Y, Higuchi S, Harigae S, Nakamura Y (2001) Identification of single nucleotide polymorphisms (SNPs) of human N-acetyltransferase genes NAT1, NAT2, AANAT, ARD1 and L1CAM in the Japanese population. J Hum Genet 46:314-319

Venter JC, Adams MD, Myers EW, Li PW, Mural RJ, Sutton GG, et al. (2001) The sequence of the human genome. Science 291:13041351 